\title{
Discussion on the Sustainable Development Pattern of Road Network Structure of Cities with a Population of 1-2 Million in China
}

\author{
Zhengmin Wen*, Zhenqiang Li, Wenshuo Luo, Yuxin FU, Juan Quan, and Guohua Zhou \\ Guilin University of Technology, Guilin, 541004, China
}

\begin{abstract}
The urban road network system is the main carrier of urban traffic. The constraint factor that urban road network has on traffic leads to major urban traffic problems. In this paper, a questionnaire survey and the Delphi method are determined to determine the case cities with a population of 1-2 million at home and abroad. Literature research and comparative, analytical, and inductive research methods are used to compare, analyse, and evaluate the advantages and disadvantages of the road network structure of the case cities, to summarize the development law of the urban road network, and to propose a sustainable development structure model for each development stage of urban road network: the mode made of ring freeway + trunk street (the embryonic stage), the mode made of ring freeway + expressway + trunk street (the incubation stage), the mode made of outer ring freeway + outer ring expressway and radial expressway + trunk street (the mature stage). The innovative point of this paper is to put forward a sustainable development pattern for the road network structure of cities with a population of 1-2 million in China.
\end{abstract}

\section{Introduction}

Sustainable development of urban transportation has become one of the key elements of the overall sustainable development of the city ${ }^{[1]}$, and road traffic is the most important way of urban transportation. Many studies have shown that the development law of urban road network structure is closely related to urban scale, urban nature, social and economic development level, and reflects the development stage of the city to a certain extent ${ }^{[2]}$. With the change of urban development direction, urban structure changes accordingly. The structure of the urban road network is related to the smoothness and convenience of people flow and logistics in each functional area outside and inside the city, and it has a direct impact on people's daily work, life and many other aspects ${ }^{[3]}$. Whether the structure is reasonable and in line with the development requirements of the times is related to the sustainable development of the city ${ }^{[4]}$, and more directly related to the convenience of our work and life. Therefore, it is of great significance to study the sustainable development pattern of urban road network structure.

At present, there are 656 cities in China, including 157 cities with a permanent population of less than 0.5 million, 256 cities with a population of 0.5-1 million, 159 cities with a population of 1-2 million, 31 cities with a population of 2-3 million, and 53 cities with a population of more than 3 million $^{[5,6,7]}$. In the next 10 years, China will be in the rapid development period of urbanization, including the extension type and connotation type. On the one hand, the scale of the urban population will expand rapidly; on the other hand, the internal structure of the city will evolve rapidly. It is predicted that there will be more than 400 cities with 1-2 million people in China by 2030, accounting for more than $60 \%$ of the total number of cities in China; meanwhile, the development of each city is basically mature, the internal structure is basically finalized, and the road network structure is also basically finalized. After 2030, the road network structure is basically stable. Therefore, it is of great theoretical significance to explore the road network structure characteristics of 1 to 2 million-population cities for guiding the planning and construction of Chinese cities.

\section{Research methods}

\subsection{Research object selection requirements and methods}

From the perspective of the formation of the existing road network of Chinese cities and the development trend of the future urban scale, the selected research objects meet the following requirements: firstly, all the cities are in the Middle and Eastern China with relatively higher economic development level and relatively perfect road system, therefore, these cities are of relatively typical significance for the study of the urban road network structure of China; secondly, the urban population scales are 1-2 million.

In the selection of research objects, the questionnaire method and Delphi method are used. First of all, according to the above requirements, 50 cities with a population of

\footnotetext{
* Corresponding author: 812475701@qq.com
} 
1-2 million are selected from the cities in East China, Central China, South China, North China, Southwest, Northwest, and Northeast China. The urban population, built-up area, GDP, and other indicators are listed in the form of a questionnaire. Then, 16 research objects are finally determined by the Delphi method. The results are as follows:

East China: Yangzhou (2.12 million), Fuzhou (2.1 million), Xiamen (1.55 million), Hefei (2 million $)^{[5,6,7]}$

Central China: Luoyang (1.8 million), Zhuzhou (1.21 million $)^{[5,6,7]}$

Northeast China: Jilin (1.5 million), Anshan (1.73 million $)^{[5,6,7]}$

South China: Zhuhai (1.12 million), Shantou (1.98 million), Huizhou (1.32 million), Haikou (1.54 million), Guilin (1.11 million) ${ }^{[5,6,7]}$

North China: Handan (1.63 million), Baotou (1.7 million), Hohhot (1.32 million) ${ }^{[5,6,7]}$

\subsection{Data acquisition method}

After the research objects are determined, the basic information of urban traffic status, including the main transport mode, the road section, the road grade structure, and the other contents, will be sorted out first through the access to relevant urban literature and network search. Secondly, the traffic data of the urban road network map, the latest urban map, or urban traffic map in different periods of each city will be collected to compare the urban road network development laws. At the same time, combined with the methods of a field survey on some cities, the field survey is carried out on the main areas and road sections of the city to confirm the urban road network and public service facilities.

\subsection{Compilation method of working base map}

In this paper, the urban maps or urban traffic maps of the selected cities are taken as the base maps, and the external freeway and main urban roads (expressways and trunk streets) of the city are shown in the figures, and the external traffic facilities such as bus stations and railway stations, and tourist attractions, as well as the related elements of the public service facilities such as shopping malls, stadiums, squares, cinemas, libraries and exhibition halls are shown in the figures in the form of lines and points $^{[8]}$, and finally form the work base maps of the research.

\subsection{Research methods}

This paper mainly uses the literature research method to study the theory and the latest literature about the road network structure. Combined with the research methods of comparison, analysis, and induction, it compares and analyzes the road network of different cities at home and abroad, evaluating the advantages and disadvantages of the road network structure, summarizing the development laws of the urban road network, and puts forward the sustainable development pattern of China's cities' reasonable road network structure according to the actual conditions.

\section{Research process and results}

\subsection{Current mode of the urban road network with a population-scale of 1-2 million in China}

From the perspectives of urban external traffic, traffic between different urban functional areas, the connection between urban functional areas and major large-scale public service facilities, study the above urban road network structure, and the sustainable development pattern can be summarized as the following types (Table 1):

1) Ring freeway + ring and radial trunk streets. The city roads have formed a relatively perfect ring plus radial road network structure, and the outer-ring freeway has fully formed. Different urban functional areas are connected by ring trunk streets, and the large-scale public service facilities such as shopping malls, gymnasiums, cinemas, exhibition centers are connected by urban trunk streets. The city interior areas are connected with the entrance and exit of the freeway through the radial trunk streets. Through research, it is found that the roads between different functional areas and from the functional areas to large-scale public facilities are blocked, the burden of the main roads is heavy, and there is no connection between the expressway and the urban main roads; the connection between the urban main roads and the external traffic facilities is not enough, and there is no connection by the expressways, so it takes a lot of time to arrive at the railway station and the bus station in each area of the city; and the expressway system connecting the entrances and exits of the ring freeway has not been established yet. Fuzhou, Hefei, Handan, Luoyang, Guilin are the type of cities.

2) Semi-ring freeway + ring and radial trunk streets. The rudiment of the ring plus the radial road network has gradually appeared. The different urban functional areas are connected by weak ring trunk streets with each other, and the large-scale urban public facilities are interpenetrated on both sides of the main roads in the central area of the city. In this kind of city, the freeway appears semi-ring form in the periphery of the city. The trunk street extending from the urban center to both sides is connected with the entrance and exit of the freeway, and the urban road in the open area enters the freeway through the ring main road. For the urban road network of semiring freeways, the convergence of the inside and outside urban transport has increased the burden of the urban ring roads, and there is no necessary expressway connection between urban roads and freeways; the ring trunk street connection between different urban functional areas is weak, and the road connection between urban large public facilities and different urban functional areas is blocked, and there is no expressway connection with each other. Zhuzhou, Huizhou, Anshan are the type of cities. 
Table1. Classification of urban road network structure in China

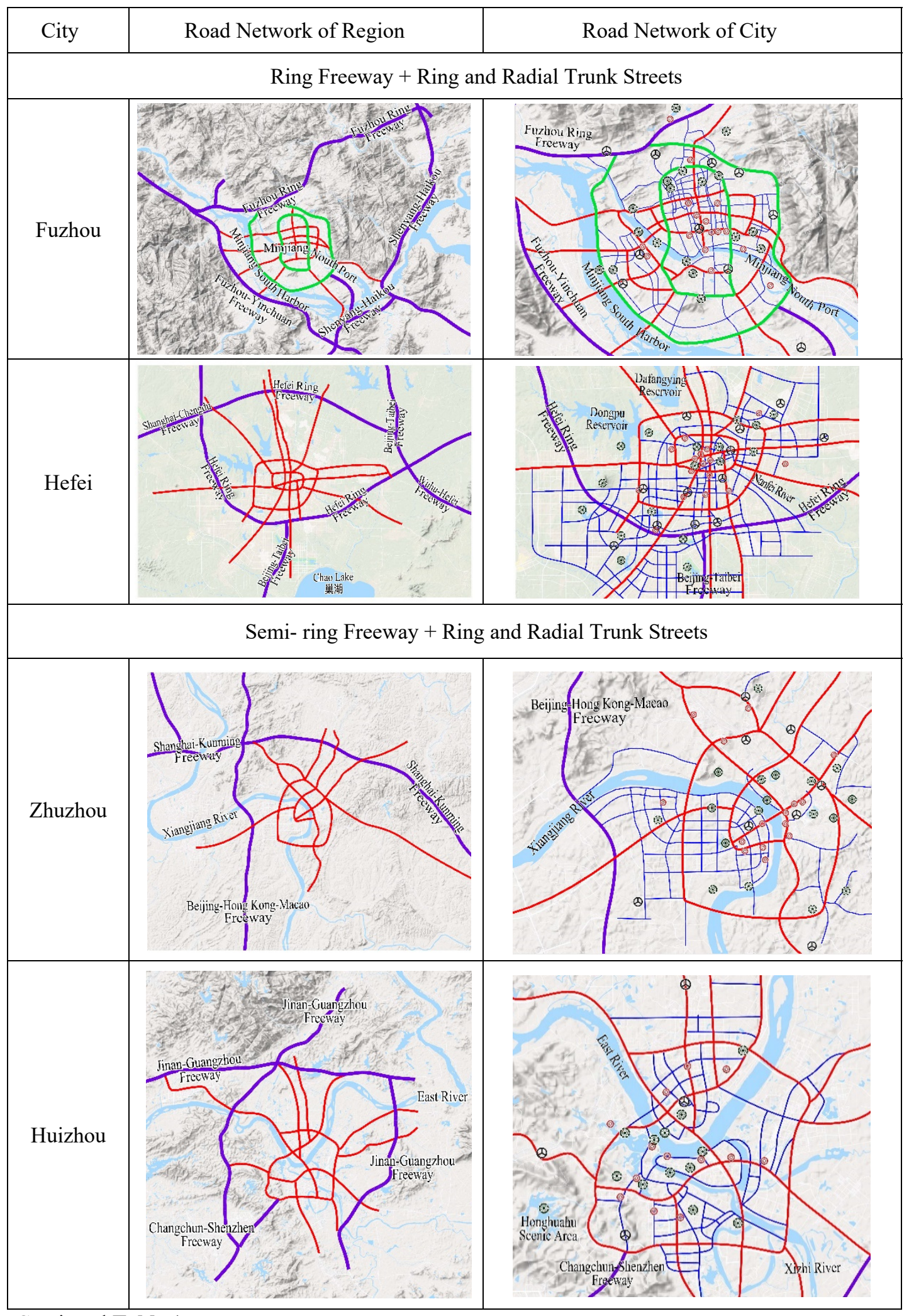

Continued Table 1.

\begin{tabular}{|c|c|c|}
\hline City & Road Network of Region & Road Network of City \\
\hline \multicolumn{2}{|c|}{ Circumscribed Freeway + Ring and Radial Trunk Streets } \\
\hline
\end{tabular}




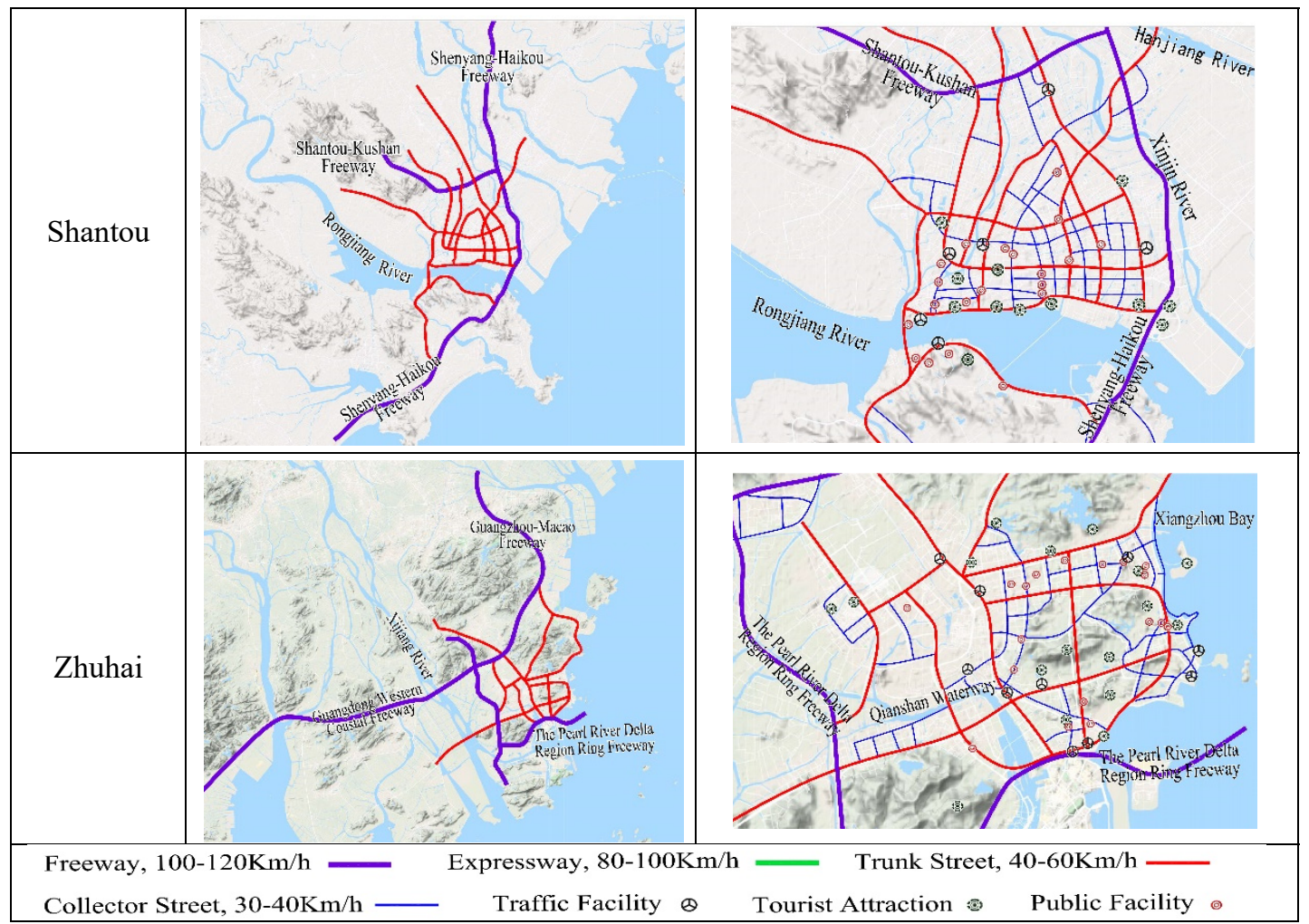

Sources: The working background based on http://ditu.google.cn/

3) Circumscribed freeway + ring and radial trunk streets. In this kind of coastal city, due to the influence of geographical locations, the freeways are all tangent to one side of a specific city. For coastal cities, the design standard of urban trunk streets is high, and urban public service facilities are organically distributed in the urban main roads network, and the roads between the different urban functional areas are generated in accordance with the city's shape. In such coastal cities, the connection of urban external traffic is only realized through the connection between urban roads and freeways on one side. Therefore, there is a lack of necessary expressway connection between them. The connections of trunk streets between different urban functional areas, and between large-scale urban public service facilities, are not enough, and there is a lack of expressway connection. In addition, considering that there should be a good connection between roads and ports, we should establish an expressway system to improve intermodal transport efficiency, such as Shantou, Zhuhai, Haikou, etc.

Based on the study of the urban road network structure characteristics with a population scale of 1-2 million in China, it is found that the road network structure types of cities with similar scale are quite different, while the road network structures of cities with different scales also have certain commonalities. From the perspective of the evolution of urban spatial structure and the development of urban road system, this phenomenon is closely related to three factors that affect the development and evolution of urban road system: the location of the city in the region, the layout of urban land, and the urban transport system. For example, Fuzhou, as the political, economic and cultural center of Fujian Province, as well as an important transport hub city in the province, has formed a road network structure of ring freeway + ring and radial trunk streets; while Shantou, a coastal city with a similar scale, has a fan-shaped urban land layout, thus forming an urban road network with fan-shaped roads as the skeleton.

\subsection{Analysis on the road network structure of cities with a population of 1-2 million in China}

Combined with the above research on the classification of the road network structure of cities with a population-scale of 1-2 million in China, the paper summarizes the characteristics of the urban road network structure of China's cities with a population-scale of 1 million, 1.5 million and 2 million respectively.

For a city with a population of about 1 million, due to the influence of the city scale and layout, the urban roads are arranged along with the public facilities, and the urban road network is relatively simple, which mainly presents a single ring plus cross-road network structure. The crossroads converge in the urban center area internally, and are connected with the freeway entrance and exit externally. This road network mode has certain limitations. The cross main road not only bears the internal traffic of the city, but also bears the connecting function between the internal and external traffic of the city, which is not conducive to the development of urban traffic.

The city with a population of about 1.5 million, with the expansion of the city scale, the urban road network gradually evolved into a ring plus radial structure, and the ring freeway gradually took shape; the radial main roads 
connect the ring road and radiate outward to connect the ring freeway. Urban trunk streets and collector streets form the main framework of urban roads. This kind of road network structure has been gradually improved. The inner ring evacuates the traffic in the central area of the city, and the outer ring separates the internal and external traffic, and reaches the entrance and exit of the freeway through the main roads between the ring roads. However, the ring roads and the main roads are mainly the trunk streets and the collector streets, the level of the roads was relatively low, resulting in the insufficient connection of the roads.

Urban roads of cities with a population of about 2 million, have established a more mature road network system. The road network structure of the ring plus the radial expressways will be gradually formed. Through the ring plus the radial expressways, the traffic flow in the central area of the city can quickly enter and leave the city, and each area can reach the external transportation facilities and large-scale public service facilities, such as railway station and bus station, and the different functional areas can also quickly contact with each other. This kind of road network structure mode has been comparatively mature. The establishment of expressway system not only meets the rapid connection of urban internal traffic, but also makes the internal and external traffic separate from each other, and gradually builds a convenient, fast, efficient and safe urban traffic system ${ }^{[9]}$.

The road network structure of cities with a population of 1-2 million in China has begun to take shape in a ring and radial way. However, because of some problems existing in the road network structure of most cities, it is proposed that in the planning stage of urban road network adjustment, the layout principles of urban expressways, trunk streets, collector streets, traffic and living roads, should be defined, and the quick connection between the urban functional groups as well as between the urban public service facilities should be considered, and the fast tracks inside and outside cities should be established. Besides, investigate the current situation, and forecast \& analyse the future of the passenger flow, vehicle flow, passenger flow and cargo flow of each main road, and reserve the space of urban fast channel in combination with the actual situation and development trend of the roads and gradually build the ring roads according to the design standard of the fast tracks, and establish the fast connecting lines such as the fast tracks or the trunk streets between the rings, to play the role of "connecting" and "reaching" of the urban main roads ${ }^{[8,10]}$.

\subsection{Analysis of road network structure mode of foreign cities with a population-scale of 1-2 million}

Foreign cities generally have large-scale freeway networks, and basically have freeways around the city periphery, such as Toulouse $(935,400,2013)[11]$ in the French Republic, Munich $\left(1,300,000,2010^{[12]}\right.$ in the Federal Republic of Germany, Detroit $(1,850,000,1950$; $880,000,2013)^{[13]}$ in the United States of America, the urban road network structure presents a double ring plus radiation shape, and the urban main roads form a ring around the city center, roads radiating outward from the center of the ring road, forming the inner-ring radial road network structure; the freeway forms a loop around the city periphery, and there are radial freeways and expressways connected with the urban main road through the ring freeway, forming the outer-ring radial freeway network structure. Except for some cities such as Detroit, due to the impact of the city's geographical location, the city center is located along the banks of the Detroit River, and the urban road network structure is in the form of fanshaped radiation. The freeway and expressway form a ring around the central area of the city, together with the urban main roads, forming a road network structure radiating outward from the center of the city (Figure 1).
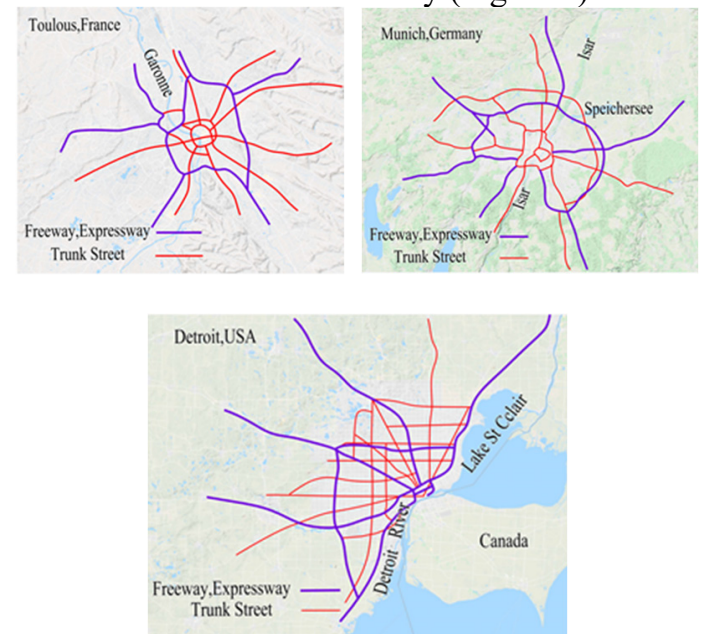

Fig1. Schematic diagram of road network structure in foreign cities. Sources: Based on the working base map provided by http://ditu.google.cn/

- The structural framework of the foreign urban road network has basically formed. Through the analysis of the structural characteristics of these mature road networks of the above cities, it is found that the road network presents certain laws.

- The freeway is closely connected with the city, and the freeways in most developed cities directly pass through the Central Business District area.

- The radial road network is relatively developed, with a large number of freeways and urban main roads radiating outwards.

- The freeway is connected unobstructedly with the urban main road, and the internal and external transport can be connected quickly.

- Shopping malls, gymnasiums and other public service facilities are also arranged on both sides of the freeway and expressway, but they are all connected through the construction of auxiliary roads to avoid interference with the freeway.

In addition, the internal road networks of city centre in western countries are mainly grid network, with uniform urban road spacing and dense distribution.

To sum up, this paper analyzes the characteristics of the foreign urban road network, and concludes that foreign urban roads have a relatively complete road grade structure, that is, the road grade system of the freeway -expressway--urban main road ${ }^{[14]}$. At the same time, the layout of the freeway, expressway, and other high-level 
roads, is more flexible and occupies a higher proportion. In terms of road network structure, it combines the structure and characteristics of the city itself. Foreign cities have formed a road network structure mode with certain characteristics, for example, ring freeway + expressway + trunk street, outer-ring freeway + outer-ring expressway, and radial expressway + urban trunk street. When the city scale and urban economy develop to a certain stage, the characteristics of the urban road network layout will be more obvious, and the road network structure system will be more perfect.

\subsection{Conception of the road network structure mode of cities with a 1-2 million population- scale in China}

The research on the urban road network structure mode at home and abroad shows that "the ring + radial road network structure" has adapted to the development of the city, and become the inevitable trend of the sustainable development of the urban road network structure in the future. Based on the analysis of the road network structure of domestic research objects, three structure types of the urban road networks in China are found: ring freeway + ring and radial trunk street, semi-ring freeway + ring and radial trunk street, and circumscribed freeway + ring and radial main road. Chinese cities have already presented the ring and radial trunk street network, and there are freeways to establish connection with cities. However, compared with the foreign urban road network structure mode, the construction pace of urban expressways is slow in China, and the urban road grade structure is not intact; the city has built peripheral freeways, yet has not formed peripheral ring freeways.

Referring to the development characteristics of foreign urban road network structure, this paper summarizes the urban road network structure of the city with 1-2 million people, which has gone through three development stages: the embryonic stage, the incubation stage, and the mature stage, namely ring freeway + trunk street, ring freeway + expressway + trunk street, outer-ring freeway + outer-ring expressway, and radial expressway + trunk street. Combined with the analysis of the characteristics of urban road network structure at home and abroad, it is concluded that the establishment of peripheral ring freeway has become an important link to support the development of urban road network structure; the expressway is the inevitable choice of urban road development. In the process of the development of urban road network in China, the construction of urban peripheral fast tracks should take the construction of peripheral ring freeway as the goal, while the urban internal fast tracks should build the expressway system, and reserve the land for expressway development, and finally form the ring and radial expressway system. When the urban scale develops to a specific stage, the urban road network structure will correspond to the characteristics of the urban road network at a certain stage, and gradually form the corresponding road network structure mode.

\section{Conclusions}

Base on summarizing and analysing the characteristics of the road network structure of cities with 1-2 million people at home and abroad, this paper puts forward that with the expansion of the urban population scale, the sustainable development pattern of the urban road network structure has developed from the mode of ring freeway + trunk street (the embryonic stage) to the mode of ring freeway + expressway + trunk street (the incubation stage), and then to the mode of outer-ring freeway + outer-ring expressway and radial expressway + trunk street (the mature stage). The selected case cities have certain representativeness, and the research conclusions also have certain representativeness. The conclusion of the study has certain theoretical significance for guiding the planning and construction of the road networks of 1 to 2 millionpopulation cities.

However, the urban road network structure is also related to many factors such as topography, socioeconomic development conditions, and the number of cases is limited. The above conclusions cannot be applied to all cities.

There will be many problems in the process of development from the embryonic stage, the incubation stage, and then to the mature stage. What problems will be encountered and how to solve these problems will be the subject that the author plans to study next.

\section{Acknowledgements}

This topic comes from the scientific research project of Guilin science and Technology Bureau "Guilin modern transportation system construction", project numbered 20080612.

\section{References}

1. Pengjun Zhao, The Impacts of Land Use Intensification on Urban Transport Sustainability: Theoretical Thinking from Literature Review, Urban Development Studies, 2018, 25 (9): 108-116

2. He Gao, Shumin Feng, Caixiang Guo, Characteristic Analysis of Structural Types of Urban Road Network, Forest Engineering, 2006, 22(5): 28-31

3. Fei Shi, Wei Wang, Urban Road Network Structure Analysis, Urban Planning, 2007, 31(8): 68-73

4. Yosef Rafeq Jabareen, Sustainable Urban Forms: Their Typologies, Models, and Concepts, Journal of Planning Education and Research, 2006, 26: 38-52

5. 2015 China Urban Statistical Yearbook, 2015

6. China's top 500 cities with urban population, database of China Business Industry Research Institute[DB/OL], http://www.askci.com/news/paihang/ 20161208/15072081751.html, /2016-05-15

7. Ranking of population in main urban areas of China in 2014, ranking of China's urban population (top 50) by permanent population in central urban areas 
[DB/OL], http://www.phbang.cn/city/145656.html, /2016-05-15

8. Xunchu Xu. Planning Thought of Urban Road Network System and Examples Analysis, Urban Transport of China, 2006, 4(1): 30-35

9. Pengfei Yang, Haodong Xie. The Optimization of Changsha Road Transportation Network Featured by Urban Space, Huazhong Architecture, 2018, 36(04):102-105

10. Lvmin Shao, Research on Xuzhou Expressway Network Planning, Proceedings of the 2017 China Urban Transport Planning Annual Conference, Urban Transport Planning Academic Committee of China Urban Planning Society, China Academy Of Urban Planning and Design, 2017: 1-8

11. Toulous [DB/OL], https://baike.baidu.com/ item $/ \%$ E5\%9B $\%$ BE $\%$ E5\%8D $\% A 2 \% E 5 \% 85 \% B 9 / 15$ 68769, /2018-09-15

12. Munich [DB/OL], https://baike.baidu.com/item/\% E6\%85\%95\%E5\%B0\%BC\%E9\%BB\%91/855, /2018-09-15

13. Urban culture, Detroit [DB/OL], https://baike. baidu.com/item/\%E5\%BA $\% 95 \%$ E7\%89\%B9\%E5\% $\mathrm{BE} \% 8 \mathrm{~B} / 1660180, / 2018-09-15$

14. America Planning Association. Hierarchy of Street and Roads, Planning and Urban Design Standards, John Wiley \& Sons, Inc., 2005: 226 